\title{
An Active Contour Model Based on Adaptive Threshold for Extraction of Cerebral Vascular Structures
}

\author{
Jiaxin Wang, ${ }^{1,2}$ Shifeng Zhao, ${ }^{1,2}$ Zifeng Liu, ${ }^{1,2}$ Yun Tian, ${ }^{1,2}$ \\ Fuqing Duan, ${ }^{1,2}$ and Yutong Pan ${ }^{1,2}$ \\ ${ }^{1}$ College of Information Science and Technology, Beijing Normal University, Beijing 100875, China \\ ${ }^{2}$ Beijing Key Laboratory of Digital Preservation and Virtual Reality for Cultural Heritage, Beijing 100875, China
}

Correspondence should be addressed to Yun Tian; tianyun@bnu.edu.cn

Received 26 April 2016; Revised 8 July 2016; Accepted 17 July 2016

Academic Editor: Giancarlo Ferrigno

Copyright (C) 2016 Jiaxin Wang et al. This is an open access article distributed under the Creative Commons Attribution License, which permits unrestricted use, distribution, and reproduction in any medium, provided the original work is properly cited.

\begin{abstract}
Cerebral vessel segmentation is essential and helpful for the clinical diagnosis and the related research. However, automatic segmentation of brain vessels remains challenging because of the variable vessel shape and high complex of vessel geometry. This study proposes a new active contour model (ACM) implemented by the level-set method for segmenting vessels from TOF-MRA data. The energy function of the new model, combining both region intensity and boundary information, is composed of two region terms, one boundary term and one penalty term. The global threshold representing the lower gray boundary of the target object by maximum intensity projection (MIP) is defined in the first-region term, and it is used to guide the segmentation of the thick vessels. In the second term, a dynamic intensity threshold is employed to extract the tiny vessels. The boundary term is used to drive the contours to evolve towards the boundaries with high gradients. The penalty term is used to avoid reinitialization of the level-set function. Experimental results on 10 clinical brain data sets demonstrate that our method is not only able to achieve better Dice Similarity Coefficient than the global threshold based method and localized hybrid level-set method but also able to extract whole cerebral vessel trees, including the thin vessels.
\end{abstract}

\section{Introduction}

Cerebral vascular diseases have become the main incentives to dizziness, disability, and even death in many countries around the world, and the research for vessels arouses concern. The segmentation of cerebral vascular structures is important for the clinical diagnosis and analysis. In medical image processing field, segmentation means the extraction of anatomical structures of interest from original data $[1,2]$. Because of low contrast of images, edge blur, and structure complexity of cerebral vessels, the accurate segmentation is still a challenging task and deserves to be researched $[3,4]$.

Over the past few decades, a large number of methods for vessel segmentation have been proposed, including atlasbased techniques [5-10], machine learning techniques [11$14]$, and active contour model (ACM) $[15,16]$. A comprehensive review can be referred to in Lesage et al. work [17]. Among these techniques, the ACM has been widely applied in medical image segmentation because of its easy extensibility.
The ACM is based on geometric curve evolution theory and the essential idea of that technique is to evolve the initial curve or surface to the boundaries of target objects driven by internal forces and external forces [18]. Active contours can be implicitly presented by the level-set methods, which put original curves into higher dimensional spaces to research and are achieved in numerical computations by the Eulerian approach [19].

ACMs using level-set formulation have various forms of expression, and they are divided into three major categories: edge-based, region-based, and hybrid level-set models. In edge-based models, edges are usually generated first by an edge-detection algorithm and then using postprocessing to adjust to the final boundaries [20]. The typical edgebased model is the geodesic active contour model [21]. The model combines active contours with the computation of geodesic distance curve, and it allows to associate classical snakes based on energy minimization with geometric active contours based on the theory of curve evolution. 
A method using a new indicator (i.e., salient edge energy) to guide a given contour robustly and accurately towards the target object boundary was proposed by W. Kim and C. Kim [22]. They defined the salient edge energy by exploiting the higher order statistics on the diffusion space and embedded it into a variational level-set function. But the edge-based models are sensitive to noise and seek to oversegment an image.

Region-based models are built on using the similarity among pixels to form homogeneous regions in an image [20]. The Mumford-Shah (M-S) model is the typical technique of that, and it depends on the defined edge function based on image gradient to stop the evolution process of active contour curves. When the contour curve is closer to the boundary of a target object, the value of gradient is higher, which causes the edge function to be closer to zero, and the evolutionary curve stops at the location of boundaries [23].

Based on the M-S model, Chan and Vese proposed the famous C-V model [24]. The C-V model can detect objects whose boundaries are not necessarily defined by gradient, because the stopping term defined in the energy function depends on the gradient of an image and is instead associated with particular segmentation of the image. In addition, the authors give a numerical algorithm using finite differences. Based on the C-V model, Tian et al. [25] proposed embedding local intensity weighting and a vessel vector field into the vessel active contour model. However, the model needed to be improved to better match segment $3 \mathrm{D}$ vessels. In these methods, it is essential to reinitialize the level-set function to make it close to the signed distance function [26]. However, the periodic reinitialization is time-consuming, and it is difficult to prevent the level-set function from being too steep or flat during the evolution [27]. To solve the problem, Li et al. [28] proposed a method through embedding the penalty term for penalizing the deviation of the level-set function from a signed distance function into the energy function.

Combining region-based methods with other information, Said [29] proposed a robust level-set-based multiregion and texture image segmentation approach. Zhang et al. [30] proposed a method to associate interactively specified regions of interest with the active contour model while keeping the user interaction to the minimum. Sciolla et al. [31] proposed a multigrid level-set segmentation method based on a regionbased function, the Hellinger distance. Jiang et al. [32] used the hybrid level-set method with a nonlinear speed function to extract brain from cerebral MRI volume. Zhao et al. [33] developed a MIP-guided approach for brain vessel segmentation. They first projected the volume onto the $2 \mathrm{D}$ plane, applied an integrated active contour model to extract blood vessels from MIP images, and then projected back to the $3 \mathrm{D}$ volume. The proposed method showed satisfying segmenting results. However, their method is a little complicated with several projection and back projection operations.

In this study, we propose an ACM implemented by the level-set method in order to segment cerebral vascular structures from TOF-MRA data. We consider both the region information and edge information and combine them to characterize the energy function. A fixed gray threshold is used into the region term to represent the global information.
In addition, we embed the adaptively dynamic threshold into our model to depict local region information, which is helpful for segment more integrated vessels. To avoid reinitializing level-set function in every evolution, the penalty term proposed by $\mathrm{Li}$ et al. is extended to $3 \mathrm{D}$ and applied into our model.

The organization of this study is as follows. In Section 2, we will introduce the related works. In Section 3, the proposed segmentation methodology is depicted. Experimental validations and discussion are given in Section 4 . Section 5 concludes the paper.

\section{Related Works}

Considering both region information and boundary information and combining them to characterize the energy function is a good idea for segmenting the complex objects. Zhang et al. [34] proposed a hybrid level-set (i.e., HLS) method for segmentation of medical images. They use preset $\mu$ value which represents the lower gray-level of target object to replace $\mu_{\text {in }}$ and $\mu_{\text {out }}$ in the region term of traditional C-V model, and the geodesic active contour model is applied to represent the edge term. The definition of energy function to be minimized is defined as

$$
\varepsilon(\phi)=-\alpha \int_{\Omega}(I-\mu) H(\phi) d \Omega+\beta \int_{\Omega} g|\nabla H(\phi)| d \Omega
$$

where $I$ is the image to be segmented, $\Omega$ is the image domain, $\alpha$ and $\beta$ are weighting factors to balance the first-region term and the second-boundary term, and the zero level set of level-set function $\phi$ represents the active contour. $H(\phi)$ is the Heaviside function defined as

$$
H(\phi)= \begin{cases}1, & \text { if } \phi \geq 0 \\ 0, & \text { if } \phi<0\end{cases}
$$

Parameter $\mu$ is preseted representing the lower gray boundary of the object to be segmented, which means it will extract the object with gray higher than $\mu$. However, since the value of $\mu$ is fixed, it cannot fit the wide intensity distribution of vessels well, especially for those small thin ones.

To solve the problem, Hong et al. [35] proposed a localized hybrid level-set (i.e., LLS) method for the segmentation of $3 \mathrm{D}$ vessel images, and they calculated locally specified dynamic threshold $\mu(\mathbf{u})$ to indicate the lower bound of target object and embedded the local gray information into the region term. Defined function $\mu(\mathbf{u})$ is

$$
\mu(\mathbf{u})=\frac{k * K_{\sigma}(\mathbf{u}) *[H(\phi(\mathbf{u})) I(\mathbf{u})]}{K_{\sigma}(\mathbf{u}) * H(\phi(\mathbf{u}))},
$$


where $\mathbf{u} \in \Omega, k \in[0.5,1]$ is an adjusting coefficient for preventing the active contours stopping evolution inside the target areas before reaching the boundary, and $K_{\sigma}$ is the Gaussian kernel function characterizing the intensity profile of a blood vessel cross section, such as

$$
K_{\sigma}(\mathbf{u})=\frac{1}{2 \pi \sigma^{2}} e^{-|\mathbf{u}|^{2} / 2 \sigma^{2}}
$$

which is used to convolve with the image in order to detect the main vessels. By the use of dynamic threshold $\mu(\mathbf{u})$ defined in (3), the method can segment the tiny vessels better, but it may lose some intensity information of thick parts due to dynamic $\mu(\mathbf{u})$ formulation limitations.

In any way, the above two methods both have respective pros and cons. Former preseted $\mu$ value drives the contours to enclose thick vessel boundaries with gray-levels greater than $\mu$, but it can not perform well on tiny vessels. The latter with dynamic $\mu(\mathbf{u})$ value can deal with the tiny vessels better but does not extract the thick vessels completely. Thus, in our study, we take full advantage of these two methods. Meanwhile, we also extend the penalty term proposed by Li et al. [28] from 2D segmentation to 3D application and embed it into the energy function to keep the characteristic of a signed distance function.

\section{Proposed Methodology}

3.1. Definition of the Energy Function. To tackle 3D cerebral vessel segmentation, we propose a new hybrid level-set model (i.e., NHLS model) inspired by models in $[34,35]$. To segment more integral vessels, we incorporate dynamic $\mu(\mathbf{u})$ value to the original hybrid model, and the proposed energy function is defined as follows:

$$
\begin{aligned}
\varepsilon(\phi)= & -\alpha_{1} \int_{\Omega}\left(I-\mu_{0}\right) H(\phi) d \Omega \\
& -\alpha_{2} \int_{\Omega}(I-\mu(\mathbf{u})) H(\phi) d \Omega \\
& +\beta \int_{\Omega} g|\nabla H(\phi)| d \Omega+\gamma P(\phi),
\end{aligned}
$$

where $I$ is the $3 \mathrm{D}$ volume data to be segmented, $\Omega$ is data domain, $\mathbf{u} \in \Omega, \mu_{0}$ is set based on the lower graylevel boundary of the target object, and $\mu(\mathbf{u})$ is calculated according to (3) representing the local threshold.

In (5), the four terms play different roles. The first term represents the global region information which drives the active contour curve to get close to the regions with bigger intensity value than $\mu_{0}$. The second term is used to represent local region information which adaptively adjust to the threshold to segment the local tiny parts. The role of the thirdboundary term is equivalent to the geodesic active contour model, and it encourages the contour curve to enclose the regions with high image gradient. Parameters $\alpha_{1}, \alpha_{2}$ and $\beta$ are used to balance the two region terms and one boundary term. And the fourth term is the penalty term, in which $\gamma$ is a preseted parameter controlling the effect of penalizing the deviation of $\phi$ from a signed distance function, and $P(\phi)$ is the penalty term to avoid reinitializing $\phi$ in evolution, which is defined as

$$
P(\phi)=\int_{\Omega} \frac{1}{2}(\nabla \phi-1)^{2} d \Omega
$$

The related PDE can be derived from the gradient decent flow applied to functional (5):

$$
\begin{aligned}
& \frac{\partial \phi}{\partial t}=\delta(\phi) \\
& \cdot\left[\alpha_{1}\left(I-\mu_{0}\right)+\alpha_{2}(I-\mu(\mathbf{u}))+\beta \operatorname{div}\left(g \frac{\nabla \phi}{|\nabla \phi|}\right)\right] \\
& +\gamma\left(\Delta \phi-\operatorname{div}\left(\frac{\nabla \phi}{|\nabla \phi|}\right)\right) .
\end{aligned}
$$

3.2. Implementation. Edge function $g(\cdot)$ represents the regularized gradient map used for geodesic active contour and nonlinear diffusion related to boundary feature of the image. In this study, $g(\cdot)$ is defined as

$$
g(x)=\frac{1}{\left(1+x^{2}\right)} \text {. }
$$

Function $H(\cdot)$ is the Heaviside function and the original function is not continuous; therefore, it cannot fit the smooth boundary curve of the practical object. To solve this problem, it is usual to use a kind of smooth function to replace the original one. There are various proposed smooth types of the Heaviside function. We adopt the smooth Heaviside function $H(\phi)$ as follow:

$$
H(\phi)=\frac{1}{2}\left(1+\frac{2}{\pi} \arctan \left(\frac{\phi}{\varepsilon}\right)\right) .
$$

And the definition of corresponding Dirac function $\delta(\phi)$ is

$$
\delta(\phi)=\frac{1}{\pi} \frac{\varepsilon}{\varepsilon^{2}+\phi^{2}}
$$

We use the above computations $H(\phi)$ and $\delta(\phi)$ to replace original $H(\cdot)$ and $\delta(\cdot)$ in (2) and (7), respectively.

Considering the penalty term in (7), $\Delta$ is the Laplacian operator, and

$$
\Delta \phi-\operatorname{div}\left(\frac{\nabla \phi}{|\nabla \phi|}\right)=\operatorname{div}\left[\left(1-\frac{1}{|\nabla \phi|}\right) \nabla \phi\right]
$$

has factor $1-1 /|\nabla \phi|$ as diffusion rate. If $|\nabla \phi|>1$, the diffusion rate is positive. If $|\nabla \phi|<1$, the diffusion rate is negative. 
Equation (7) can be simply written as

$$
\frac{\phi^{k+1}-\phi^{k}}{\Delta t}=\alpha_{1} M_{1}+\alpha_{2} M_{2}+\beta N+\gamma P
$$

where $\phi^{k+1}$ and $\phi^{k}$ denote the level-set function $\phi$ in $(k+1)$ th and $k$ th iterations, respectively, $\Delta t$ is the preset time step, $M_{1}$ is the global region term, $M_{2}$ is the local term, $N$ is the edge term, and $P$ is the penalty term. It is required to illustrate that $M_{1}$ is a fixed value decided by $I$ and $\mu_{0}$ is not related to $\phi$. $M_{2}, N$, and $P$ can be also expressed as $M_{2}\left(\phi^{k}\right), N\left(\phi^{k}\right)$, and $P\left(\phi^{k}\right)$, and they are affected by $\phi^{k}$. Difference equation (12) can be represented as follows:

$$
\phi^{k+1}=\phi^{k}+\Delta t\left(\alpha_{1} M_{1}+\alpha_{2} M_{2}+\beta N+\gamma P\right) .
$$

Iteration from $\phi^{k}$ to $\phi^{k+1}$ includes five steps:

(i) Compute dynamic localized threshold $\mu(\mathbf{u})$ according to $(3)$.

(ii) Compute penalty term $P$ in terms of (6).

(iii) Calculate $\alpha_{1} M_{1}+\alpha_{2} M_{2}+\gamma P$.

(iv) Update $\phi^{k}$ to $\phi^{k^{\prime}}$ using $\phi^{k^{\prime}}=\phi^{k}+\Delta t\left(\alpha_{1} M_{1}+\alpha_{2} M_{2}+\right.$ $\gamma P)$.

(v) Update $\phi^{k^{\prime}}$ to $\phi^{k+1}$ using $\phi^{k+1}=\phi^{k^{\prime}}+\Delta t \beta N$, which is achieved by the semiexplicit method. In fact, we can also use the explicit method to get $\phi^{k+1}$ directly, which plays the same role with the explicit method. However, explicit methods have limitations in time steps, and they need to set time steps small to keep methods stable. If we use explicit methods, the time steps maybe set small to make sure that the process of evolution maintains stability, which leads to time-consuming process [36]. Thus, we choose the semiexplicit method.

There is an additional problem to address that is to set the initial curve of level set. In this study, we apply Frangi's vessel enhancement algorithm into the original data and then implement the canny detection to get the fuzzy boundary of vessel. That boundary curve is used as the initial curve. By this method, it can make sure that every evolution is around the vessel region, which improves efficiency.

3.3. Outlier Removement. Since the intensity value of some nonvessel points are very close to those of vessel points, some nonvessel points (i.e., outlier) exist in the segmentation results. In order to remove the outlier points around vessels as much as possible, we need to consider the shape feature of vessels.

Eigenvalues of the Hessian matrix have been successfully used in blood vessel enhancement [37]. For a 3D volume, we assume that the eigenvalues of the Hessian matrix are sorted as $\left|\lambda_{1}\right| \geq\left|\lambda_{2}\right| \geq\left|\lambda_{3}\right|$. The ideal tubular structure in a $3 \mathrm{D}$ volume would have

$$
\begin{gathered}
\left|\lambda_{3}\right| \approx 0, \\
\left|\lambda_{3}\right| \ll\left|\lambda_{2}\right|, \\
\lambda_{1} \approx \lambda_{2} .
\end{gathered}
$$

Furthermore, in MRA images, the fact is that vessel structures are brighter than the background and the Frangi's vessel enhancement algorithm makes use of all the eigenvalues of Hessian matrix, and it can consider fully the geometric feature that the eigenvalues represent and suppress the impact of irrelevant points on vessels. They define two geometric ratios $R_{A}, R_{B}$, and $S$, respectively, as

$$
\begin{aligned}
R_{B} & =\frac{\left|\lambda_{1}\right|}{\sqrt{\left|\lambda_{2} \lambda_{3}\right|}}, \\
R_{A} & =\frac{\left|\lambda_{2}\right|}{\left|\lambda_{3}\right|}, \\
S & =\sqrt{\lambda_{1}{ }^{2}+\lambda_{2}{ }^{2}+\lambda_{3}{ }^{2}},
\end{aligned}
$$

where $R_{B}$ gets maximum for a blob structure, $R_{A}$ differentiates plane structures from line structures because in the latter situation it will be zero, and $S$ is the measure to distinguish background which will be slow because the eigenvalues are small in the background. On the basis of the three parameters, Frangi et al. define a vesselness function combining those components as follows:

$$
V_{\sigma}(S)= \begin{cases}0, & \text { if } \lambda_{2}>0 \text { or } \lambda_{3}>0 \\ \left(1-\exp \left(-\frac{R_{A}{ }^{2}}{2 \alpha^{2}}\right)\right) \exp \left(-\frac{R_{B}{ }^{2}}{2 \beta^{2}}\right)\left(1-\exp \left(-\frac{S^{2}}{2 c^{2}}\right)\right), & \text { otherwise, }\end{cases}
$$

where $\alpha, \beta$, and $c$ are thresholds of 3D vesselness function which is used to control the sensitivity of vessel enhancement filter to parameters $R_{A}, R_{B}$, and $S$.

As multiscale eigenanalysis of local Hessian operators can enhance local rod-like shapes of varying radii. The value of vesselness function is between 0 and 1 . If objects are tubular structures, vesselness function $V_{\sigma}(S)$ is close to 1 . For an ideal tubular structure, $R_{A} \approx 1, R_{B} \approx 0$. It is noticed that when $R_{B} \approx 0$, the second term in (16) is approximately equal to 1 . However, when $R_{A} \approx 1$, the value 


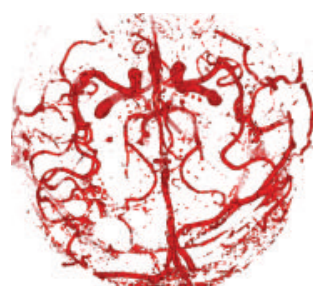

(a)

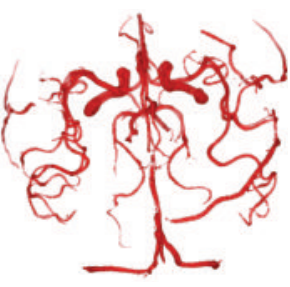

(b)

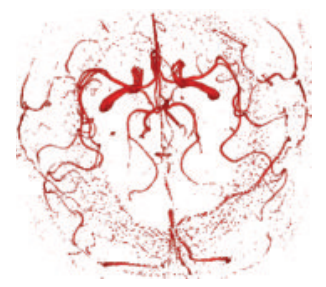

(c)

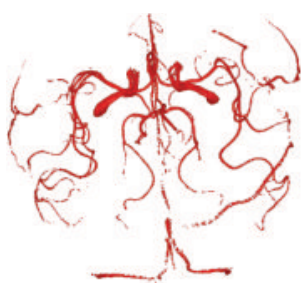

(d)

FIGURE 1: Outlier removement. NHLS segmentation before (a) and after (b) outlier voxels are eliminated with the connectivity filter and LLS segmentation before (c) and after (d) the connectivity filter.

of the first term in (16) has slight gap with 1 . In order to make $V_{\sigma}(S)$ approximately equal 1 , we take advantage of function $\tan (\pi / 2) x$. When $x \approx 1, \tan (\pi / 2) x$ approaches positive infinity, and $\exp (-\tan (\pi / 2)(x))$ is approximately equal to 0 , so $(1-\exp (-\tan (\pi / 2)(x)))$ is closer to 1 than before. Thus, in order to enhance the tubular structures to a larger extent, we modified the vesselness function as follows:

$$
V_{\sigma}(S)= \begin{cases}0, & \text { if } \lambda_{2}>0 \text { or } \lambda_{3}>0 \\ \left(1-\exp \left(-\frac{\tan ^{2}\left((\pi / 2) R_{A}\right)}{2 \alpha^{2}}\right)\right) \exp \left(-\frac{\tan ^{2}\left((\pi / 2) R_{B}\right)}{2 \beta^{2}}\right)\left(1-\exp \left(-\frac{S^{2}}{2 c^{2}}\right)\right), & \text { otherwise. }\end{cases}
$$

Considering some available information that can be used to help remove noise may be lost during the process of segmentation; therefore, the algorithm is first used onto the original data to obtain the enhancement vessel structure instead of being applied directly onto the segmentation result. Then, we use the vessel structure to guide the elimination process of nonvessel outlier points in the segmentation result.

\section{Experimental Results and Discussion}

Experiments in extracting cerebral vessels have been conducted on 10 TOF-MRA data sets which were acquired from Navy General Hospital. The 4 sets of data (Data 1, Data 2, Data 3, and Data 4) analyzed in this paper are with the size of $512 \times 512 \times 216$ voxels, the resolution of $0.39 \times 0.39 \mathrm{~mm}^{2}$, and a slice thickness of $1.2 \mathrm{~mm}$. The experiments are implemented on a computer with Intel ${ }^{\circledR}$ Core $^{\mathrm{TM}}$ i5-4590 CPU 3.30 GHZ CPU, 12.0 G RAM, and Windows 7 operating system. The parameters used are as follows: $\Delta t=2.0, \alpha_{1}=\alpha_{2}=$ $0.003, \beta=0.02, \gamma=1.0, \varepsilon=1.0$.

4.1. Comparisons with the HLS and LLS Model. As Figures 1(a) and 1(c) suggest, TOF-MRA is sensitive to fat tissues which would shutter the blood vessels. A circle of points on the top of the head is introduced in the segmented volume due to similar intensity value between tissues and blood vessels. To eliminate them, the volume is processed with an automatic connectivity filter. We first perform the Frangi's vessel enhancement method onto the original MRA data. Then, we preserve the points in our segmentation result that the first step obtained and then start regional growth algorithm using vessel connectivity. Figures $1(\mathrm{~b})$ and $1(\mathrm{~d})$ present the results after applying such a filter to LLS model and our NHLS model.

All the three methods have been experimented with 10 data sets. Results of the three tests are depicted in Figure 2. The first column of Figure 2 shows the MIP images. The second column shows the segmentation results by HLS model. The third column shows the segmentation results by LLS model. The last column shows the results segmented by our NHLS model.

As for HLS model, through analyzing the histogram of the data set, we notice that the intensity value of cerebral vascular structures is approximately higher than 200. But, there exist differences among different parts of vessels, and for some of them the intensity value may be between 150 and 200 . In our experiments, we set low intensity value $\mu_{0}$ of the three level-set method to be 200; it will extract the vessels with intensity higher than 200 and those lower than 200 will not be extracted as well. The segmentation result is shown in the second column.

As we can see, the segmentation result of cerebral vascular structures is not ideal that it only extracts the large artery structures of vessels but loses many tiny vessel branches. In that method, key parameter $\mu_{0}$ is predefined to be 200 which means it is unable to extract the small branches with intensity lower than 200. On the other hand, predefined $\mu_{0}$ is a global threshold; however, the intensity value of different vessel branches is inhomogeneous and has some differences. Therefore, it is essential to consider the local features.

As for LLS model, which is an improved method of HLS model replacing predefined $\mu_{0}$ with dynamic $\mu(\mathbf{u})$, dynamic $\mu(\mathbf{u})$ is the automatically computed local threshold. The definition of $\mu(\mathbf{u})$ is achieved by the Gaussian kernel function modeling the intensity of a blood vessel cross section. 

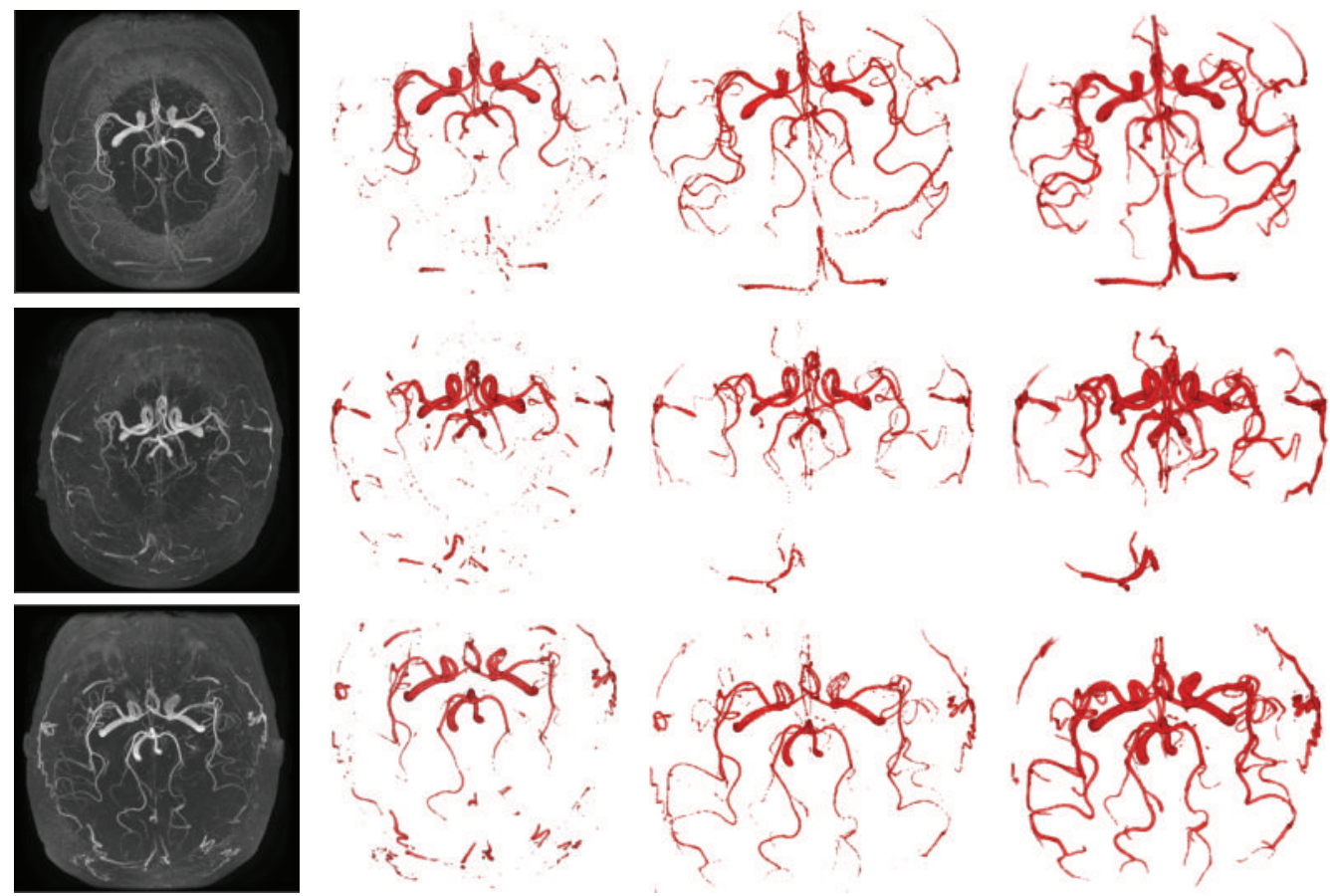

FiguRE 2: The result of cerebral vascular structures segmented by three models. Each row relates to one patient: the first column represents the MIP images. The second column shows the segmentation result of HLS model; the third column shows the segmentation result of LLS model after noise voxels are eliminated with the connectivity filter; the last column shows the segmentation of NHLS model after noise voxels are eliminated with the connectivity.

Deviation $\sigma$ of Gaussian kernel is 3.24 in our experiment. The segmentation result is in the third column.

It is noticed that the segmentation result of the second method can extract not only the thick artery structures of vessels but also the tiny branches. On one hand, because the vessel branches are very complex and intensity inhomogeneity occurs in vessel structures, threshold $\mu(\mathbf{u})$ dynamically calculated can characterize the local information of vessels better. On the other hand, using dynamic thresholds representing the lower bound of vessels can consider the regional information better and the segmentation results are more integral than the original one.

However, compared with HLS, the regions of the thick vessels extracted by LLS are not brighter, which means the segmentation result of the thick vessels is not integrated. The defect is caused since the LLS model pays more attention to local and tiny information and neglects some global information, and the segmentation result includes some irrelevant points with the similar intensity value to vessels around the vessels.

The proposed method in this paper is inspired by the HLS model and the LLS model. The result is in the last column. Our model combines the global threshold information with the localized threshold information. We analyze the histogram of data and find that the intensity value of vessels is approximately higher than 200; therefore we set global threshold $\mu_{0}$ to be 200 which means it will extract the target regions with intensity value larger than 200 . By embedding the global threshold into the energy function, we define and extract the thick main artery structures of vessels better. In addition, we conceive a dynamic threshold through the role of the Gaussian kernel function, which is used to characterize the local intensity information of vessels. The local thresholds segment the tiny vessels from background more completely.

To highlight advantages of our approach, Figure 3 presents some details of Data 1 . The first row is the segmentation result, and the second row and third row are the amplified spatial details corresponding to the local regions (marked with the blue boxes). The details show that the result of the HLS model loses many surrounding branches, the LLS model segments tiny branches, but branches are not continuous. The last column is the result segmented by NHLS which extracts those branches, at the same time, it is more integrated. In addition, the thick structures are hollow segmented by LLS with dynamic threshold, and our method solves the defect.

Besides the visual inspection, we also evaluate the segmentation accuracy using the Dice Similarity Coefficient (DSC), a widely used metric to evaluate segmentation algorithms for different medical image modalities [38]. Radiologists are invited to segment four sets of MRA data, and the segmentation results are as the ground truth. We, respectively, count the voxel numbers of results segmented by HLS, LLS, and our NHLS model and the voxel numbers of corresponding ground truths. The DSC is defined as

$$
\mathrm{DSC}=2 \times \frac{N\{M \cap G\}}{N\{M\}+N\{G\}} \times 100,
$$



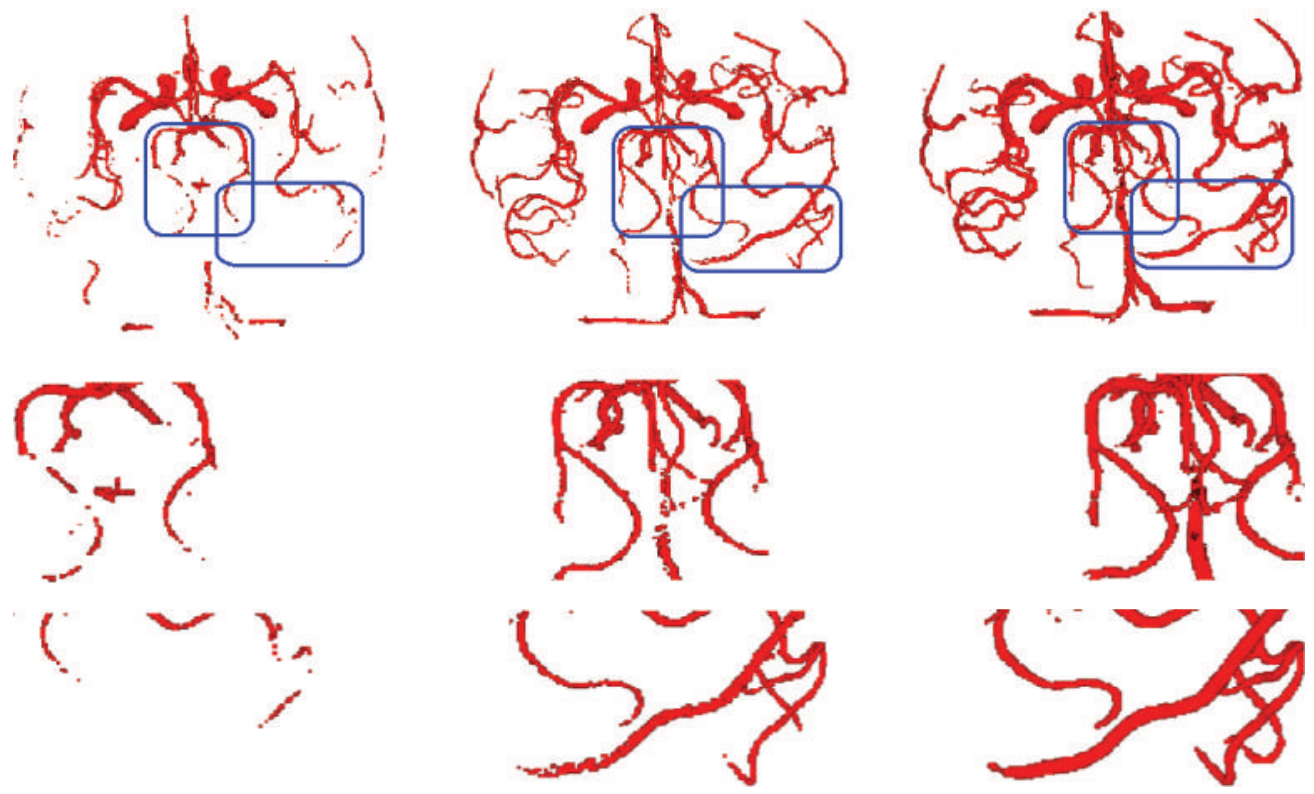

FIgURE 3: Some details. The first row represents the segmentation results by HLS, LLS, and NHLS. The second and third row are the amplified spatial details corresponding to the local region, respectively (marked with the blue boxes).

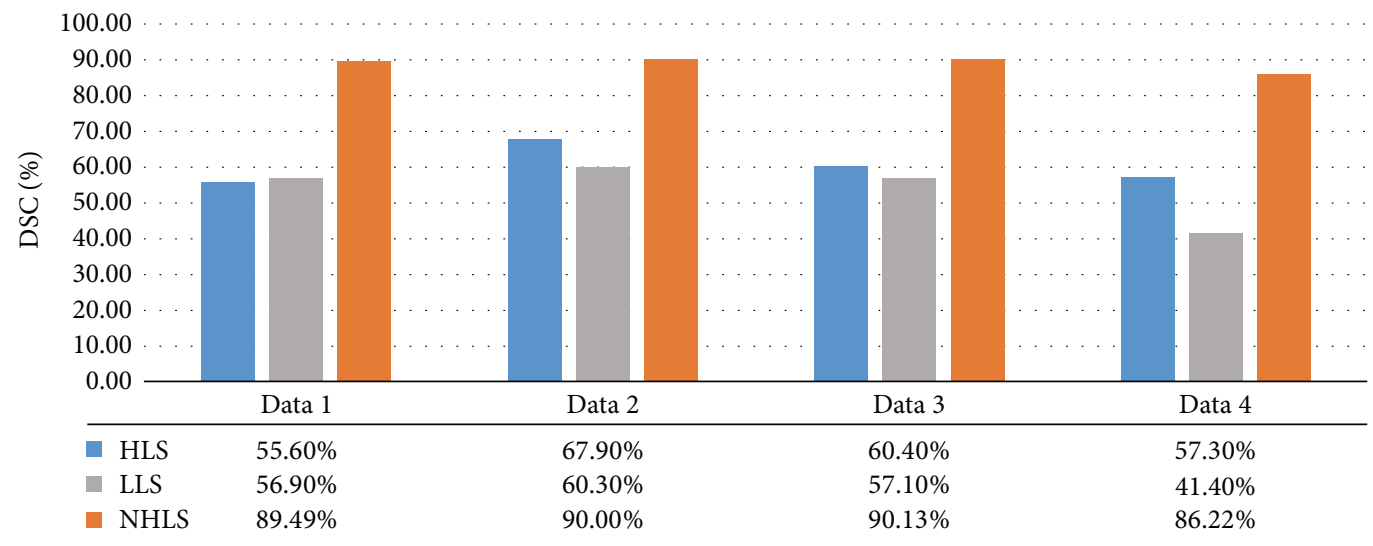

FIGURE 4: The DSC of four sets of data using three methods.

where $M$ and $G$ are the segmentation results and ground truths and $N$ is the voxel number. And $\cap$ denotes the set cardinality. It has value 1 when $M$ and $G$ are equal and 0 when they do not share any voxel.

Figure 4 summarizes the average DSC for three methods. Three observations can be made from Figure 4. First, the DSC achieved by our method is over $80 \%$ for most cases. This might be because parts of the vessel were not highlighted due to the vascular disease causing disconnection among voxels in the spatial domain. Second, the average DSC of our method is $29.7 \% \sim 44.8 \%$ higher than that of LLS. We think that is mainly due to not ideal segmentation of main thick vessels. Third, the average DSC of our method is $22.1 \%$ 33.9\% higher than that of HLS method. We believe HLS model's poor performance is mainly due to the static intensity threshold. Although we could manually select the most suitable threshold value for evolution, it remains challenging to distinguish low contrast vessel from background.

4.2. Sensitivity Analysis for the Parameters. The corresponding parameters of the above experiments are $\alpha_{1}, \alpha_{2}, \beta, \Delta t, \gamma$, and $\varepsilon$. Among them, three parameters $\alpha_{1}, \alpha_{2}$, and $\Delta t$ have more effects on the segmentation results. Parameters $\alpha_{1}$ and $\alpha_{2}$ are the weight coefficients of the two region terms, and they balance the roles between the global grayscale and the local information. By our test, when $\alpha_{1}$ equals $\alpha_{2}$, which means the two region terms play the same role, our segmentation results are better. The test on Data 1 is as shown in Table 1 . About $\Delta t$, we reference the selection of time step in [28], which considers both the speed of evolution and the error in the boundary location, and it concludes that time step $\Delta t$ usually is set smaller than 10 . In our experiments, if the time 
TABLE 1: Corresponding parameters $\alpha_{1}, \alpha_{2}$.

\begin{tabular}{lccccc}
\hline$\alpha_{2} / \alpha_{1}$ & 0.25 & 0.5 & 1 & 2 & 4 \\
\hline DSC (\%) & 82.71 & 85.12 & 89.49 & 84.00 & 80.49 \\
\hline
\end{tabular}

step is bigger, the evolution can be speeded up; however, there exist more nonvessel points in the segmentation result, which affects the accuracy. $\Delta t=2.0$ is a tradeoff and suitable for this study.

\section{Conclusions}

We have introduced a new hybrid method for the automatic segmentation of cerebral vessels based on an active contour model. The joint energy terms of static and adaptive dynamic kernel within the level-set framework allow for the extraction of thick and thin vessels as well. We have evaluated our method on 10 data sets showing that approximately $80 \%$ of DSC are required, and the method performs comparably better than the other two algorithms. Our future work includes acceleration of the current method and further accuracy improvement through vascular compartment recognition.

\section{Competing Interests}

The authors declare that they have no competing interests.

\section{Acknowledgments}

This work is supported by the National Science Foundation of China (Grant no. 61472042), the Beijing Natural Science Foundation (Grant nos. 4152027 and 4152028), the Fundamental Research Funds for the Central Universities (no. 2015KJJCB25), and the Program for New Century Excellent Talents in University (NCET-13-0051).

\section{References}

[1] H. Gao, Segmentation of cell structures in fluorescence confocal microscopy images [Ph.D. thesis], University of Central Lancashire, Preston, UK, 2013.

[2] A. Rusu, D. I. G. Stillfried, D. Institutsdirektor, and G. Hirzinger, Segmentation of bone structures in magnetic resonance images (mri) for human hand skeletal kinematics modelling [Ph.D. thesis], German Aerospace Center, 2011.

[3] C. Bhole, C. Pal, D. Rim, and A. Wismüller, "3D segmentation of abdominal CT imagery with graphical models, conditional random fields and learning," Machine Vision and Applications, vol. 25, no. 2, pp. 301-325, 2014.

[4] D. Zarpalas, P. Gkontra, P. Daras, and N. Maglaveras, "Accurate and fully automatic hippocampus segmentation using subjectspecific 3D optimal local maps into a hybrid active contour model," IEEE Journal of Translational Engineering in Health and Medicine, vol. 2, pp. 1-16, 2014.

[5] H. A. Kirisli, M. Schaap, S. Klein et al., "Fully automatic cardiac segmentation from 3D CTA data: a multi-atlas based approach," in Proceedings of the Medical Imaging 2010: Image Processing, vol. 7623 of Proceedings of SPIE, p. 762305, San Diego, Calif, USA, March 2010.
[6] P. Aljabar, R. A. Heckemann, A. Hammers, J. V. Hajnal, and D. Rueckert, "Multi-atlas based segmentation of brain images: atlas selection and its effect on accuracy," NeuroImage, vol. 46, no. 3, pp. 726-738, 2009.

[7] M. B. Cuadra, C. Polio, A. Bardera, O. Cuisenaire, J.-G. Villemure, and J.-P. Thiran, "Atlas-based segmentation of pathological MR brain images using a model of lesion growth," IEEE Transactions on Medical Imaging, vol. 23, no. 10, pp. 1301-1314, 2004.

[8] B. Landman and S. Warfield, "Miccai 2012 workshop on multiatlas labeling," in Proceedings of the Medical Image Computing and Computer Assisted Intervention Conference: MICCAI Grand Challenge and Workshop on Multi-Atlas Labeling Challenge Results, April 2012.

[9] H. Wang, J. W. Suh, S. R. Das, J. B. Pluta, C. Craige, and P. A. Yushkevich, "Multi-atlas segmentation with joint label fusion," IEEE Transactions on Pattern Analysis and Machine Intelligence, vol. 35, no. 3, pp. 611-623, 2013.

[10] A. J. Asman and B. A. Landman, "Non-local statistical label fusion for multi-atlas segmentation," Medical Image Analysis, vol. 17, no. 2, pp. 194-208, 2013.

[11] S. Powell, V. A. Magnotta, H. Johnson, V. K. Jammalamadaka, R. Pierson, and N. C. Andreasen, "Registration and machine learning-based automated segmentation of subcortical and cerebellar brain structures," NeuroImage, vol. 39, no. 1, pp. 238 247, 2008.

[12] X. Ren and J. Malik, "Learning a classification model for segmentation," in Proceedings of the 9th IEEE International Conference on Computer Vision, pp. 10-17, IEEE, Nice, France, October 2003.

[13] A. A. Othman and H. R. Tizhoosh, "Segmentation of breast ultrasound images using neural networks," in Engineering Applications of Neural Networks, pp. 260-269, Springer, New York, NY, USA, 2011.

[14] A. P. Bradley, "The use of the area under the ROC curve in the evaluation of machine learning algorithms," Pattern Recognition, vol. 30, no. 7, pp. 1145-1159, 1997.

[15] A. K. Mishra, Decoupled deformable model for 2D/3D boundary identification [Ph.D. thesis], University of Waterloo, 2010.

[16] Y. Tian, F. Duan, M. Zhou, and Z. Wu, "Active contour model combining region and edge information," Machine Vision and Applications, vol. 24, no. 1, pp. 47-61, 2013.

[17] D. Lesage, E. D. Angelini, I. Bloch, and G. Funka-Lea, "A review of $3 \mathrm{D}$ vessel lumen segmentation techniques: models, features and extraction schemes," Medical Image Analysis, vol. 13, no. 6, pp. 819-845, 2009.

[18] A. Valsecchi, P. Mesejo, L. Marrakchi-Kacem, S. Cagnoni, and S. Damas, "Automatic evolutionary medical image segmentation using deformable models," in Proceedings of the IEEE Congress on Evolutionary Computation (CEC '14), pp. 97-104, IEEE, Beijing, China, July 2014.

[19] S. Osher and J. A. Sethian, "Fronts propagating with curvaturedependent speed: algorithms based on Hamilton-Jacobi formulations," Journal of Computational Physics, vol. 79, no. 1, pp. 1249, 1988.

[20] A. J. Judah, On adaptive image segmentation of remotely sensed imagery [Ph.D. thesis], York University, Toronto, Canada, 2014.

[21] V. Caselles, R. Kimmel, and G. Sapiro, "Geodesic active contours," International Journal of Computer Vision, vol. 22, no. 1, pp. 61-79, 1997. 
[22] W. Kim and C. Kim, "Active contours driven by the salient edge energy model," IEEE Transactions on Image Processing, vol. 22, no. 4, pp. 1667-1673, 2013.

[23] D. Mumford and J. Shah, "Optimal approximations by piecewise smooth functions and associated variational problems," Communications on Pure and Applied Mathematics, vol. 42, no. 5, pp. 577-685, 1989.

[24] T. F. Chan and L. A. Vese, "Active contours without edges," IEEE Transactions on Image Processing, vol. 10, no. 2, pp. 266-277, 2001.

[25] Y. Tian, Q. Chen, W. Wang et al., "A vessel active contour model for vascular segmentation," BioMed Research International, vol. 2014, Article ID 106490, 15 pages, 2014.

[26] D. Peng, B. Merriman, S. Osher, H. Zhao, and M. Kang, "A PDEbased fast local level set method," Journal of Computational Physics, vol. 155, no. 2, pp. 410-438, 1999.

[27] J. Gomes and O. Faugeras, "Reconciling distance functions and level sets," Journal of Visual Communication and Image Representation, vol. 11, no. 2, pp. 209-223, 2000.

[28] C. Li, C. Xu, C. Gui, and M. D. Fox, "Level set evolution without re-initialization: a new variational formulation," in Proceedings of the IEEE Computer Society Conference on Computer Vision and Pattern Recognition (CVPR '05), vol. 1, pp. 430-436, IEEE, June 2005.

[29] A. F. Said, Noise resilient image segmentation and classification methods with applications in biomedical and semiconductor images [Ph.D. thesis], Arizona State University, Tempe, Ariz, USA, 2010.

[30] Y. Zhang, B. J. Matuszewski, A. Histace, F. Precioso, J. Kilgallon, and C. Moore, "Boundary delineation in prostate imaging using active contour segmentation method with interactively defined object region," in Prostate Cancer Imaging. Computer-Aided Diagnosis, Prognosis, and Intervention: International Workshop, Held in Conjunction with MICCAI 2010, Beijing,China, September 24, 2010. Proceedings, vol. 6367 of Lecture Notes in Computer Science, pp. 131-142, Springer, Berlin, Germany, 2010.

[31] B. Sciolla, P. Delachartre, L. Cowell, T. Dambry, and B. Guibert, "Multigrid level-set segmentation of high-frequency 3D ultrasound images using the Hellinger distance," in Proceedings of the 9th International Symposium on Image and Signal Processing and Analysis (ISPA '15), pp. 165-169, IEEE, Zagreb, Croatia, September 2015.

[32] S. Jiang, W. Zhang, Y. Wang, and Z. Chen, "Brain extraction from cerebral MRI volume using a hybrid level set based active contour neighborhood model," BioMedical Engineering Online, vol. 12, article 31, 2013.

[33] S. Zhao, M. Zhou, Y. Tian, P. Xu, Z. Wu, and Q. Deng, "Extraction of vessel networks based on multiview projection and phase field model," Neurocomputing, vol. 162, pp. 234-244, 2015.

[34] Y. Zhang, B. J. Matuszewski, L.-K. Shark, and C. J. Moore, "Medical image segmentation using new hybrid level-set method," in Proceedings of the 5th International Conference BioMedical Visualization (MediVis '08), pp. 71-76, London, UK, July 2008.

[35] Q. Hong, Q. Li, B. Wang et al., "3D vasculature segmentation using localized hybrid level-set method," BioMedical Engineering OnLine, vol. 13, no. 1, p. 169, 2014.

[36] J. Weickert, B. M. Ter Haar Romeny, and M. A. Viergever, "Efficient and reliable schemes for nonlinear diffusion filtering," IEEE Transactions on Image Processing, vol. 7, no. 3, pp. 398-410, 1998.
[37] A. F. Frangi, W. J. Niessen, K. L. Vincken, and M. A. Viergever, "Multiscale vessel enhancement filtering," in Medical Image Computing and Computer-Assisted Interventation-MICCAI'98, pp. 130-137, Springer, Berlin, Germany, 1998.

[38] A. P. Zijdenbos, B. M. Dawant, R. A. Margolin, and A. C. Palmer, "Morphometric analysis of white matter lesions in MR images: method and validation," IEEE Transactions on Medical Imaging, vol. 13, no. 4, pp. 716-724, 1994. 


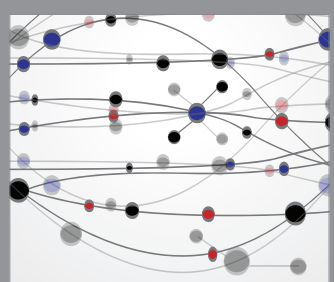

The Scientific World Journal
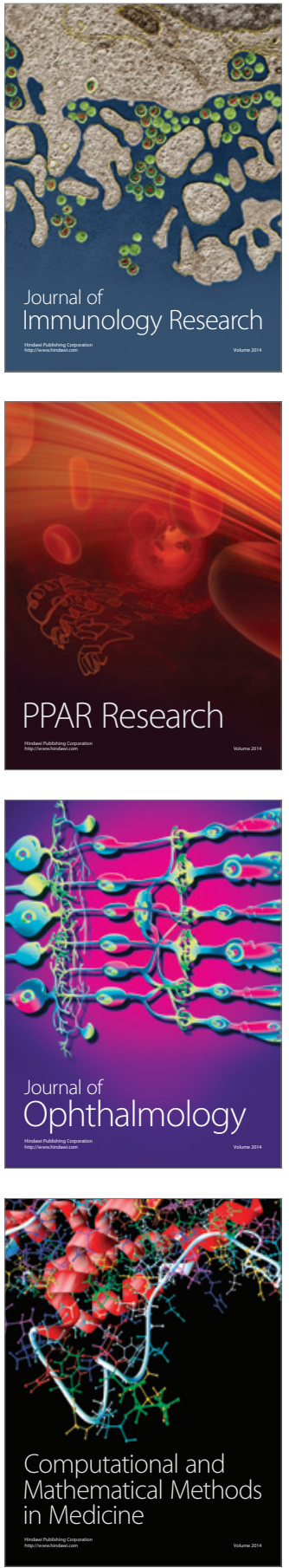

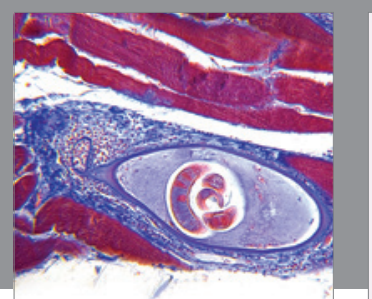

Gastroenterology Research and Practice

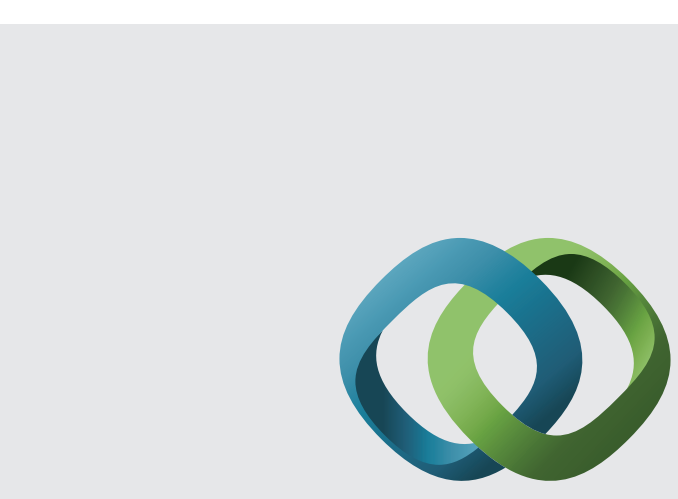

\section{Hindawi}

Submit your manuscripts at

http://www.hindawi.com
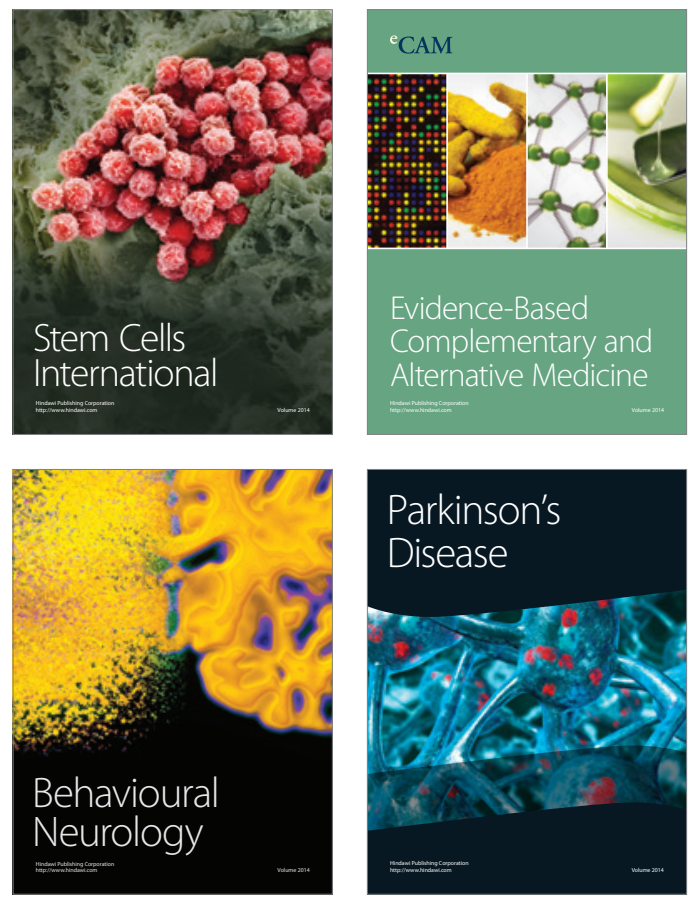
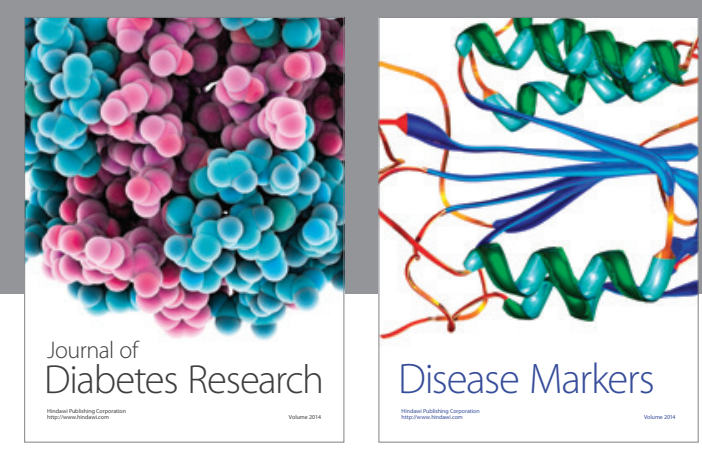

Disease Markers
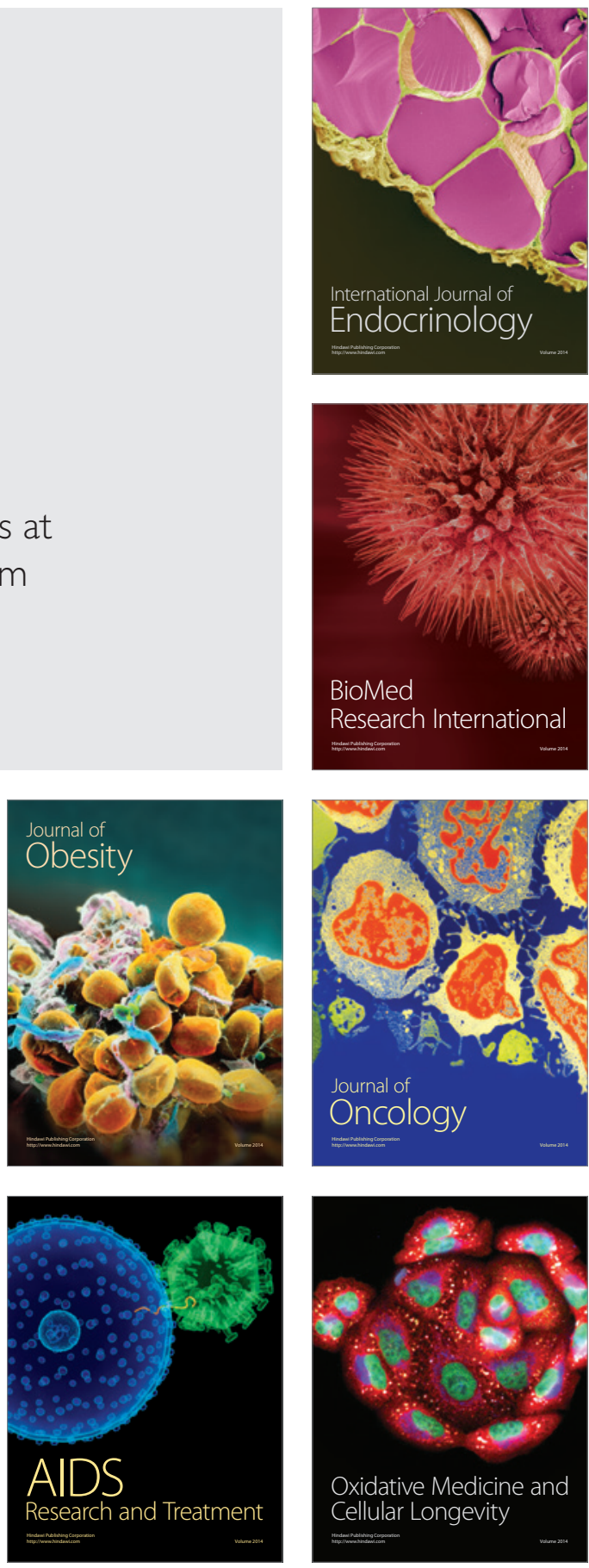